\title{
Variabilidad térmica interanual como base para el estudio del calentamiento global. El caso de la República Argentina (1982-2012)
}

\author{
Interannual termal variability as a basis for the study of global \\ warming. The case of the Argentine Republic (1982-2012)
}

\author{
Patricia Perla Snaider \\ Profesora, Universidad Nacional del Nordeste, Argentina. \\ pasnaider@hotmail.com | 0000-0003-1425-0687 \\ Oscar Luis Pyszczek \\ Profesor, Universidad de Ciencias Aplicadas y Ambientales, Colombia. \\ opysxczek@udca.edu.co $\mid \underline{0000-0003-4883-0583}$
}

Para citar este artículo: Snaider, P. P., y Pyszczek, O. L. (2020). Variabilidad térmica interanual como base para el estudio del calentamiento global. el caso de la república argentina (1982-2012). Entorno Geográfico, (20), 88-108.

https://doi.org /10.25100/eg.v0i20.10847

\section{Resumen}

El debate referido al calentamiento global del planeta ha abandonado los claustros académicos para convertirse en una certeza científica y en una creciente preocupación global visibilizada en los Objetivos de Desarrollo Sostenible de la ONU, vinculado a la acción por el clima (ODS 13). Estas circunstancias conllevan a la necesidad de investigaciones tendientes a establecer líneas de base que identifiquen las áreas de mayor riesgo climático hacia al interior de los Estados nacionales. La República Argentina es el octavo país más extenso del planeta y las consecuencias de la variabilidad térmica interanual no afectan homogéneamente a su territorio. En tal sentido, el presente artículo pretende identificar las anomalías térmicas interanuales, evidenciadas en la Argentina en el período 1982-2012 como base para la comprensión del calentamiento global. El objetivo consiste en establecer las principales áreas de anomalías térmicas mediante la técnica de análisis estadístico-cartográfico, con el fin de generar conocimientos de base acerca de los efectos de la variabilidad térmica interanual en este país sudamericano. Palabras clave: Calentamiento global, variabilidad térmica interanual, temperaturas, anomalías, Argentina.

\begin{abstract}
Abatract
The debate on global warming has left the academic cloisters to become a scientific certainty and a growing global concern visible in the UN's Sustainable Development Goals, linked to climate action (ODS 13). These circumstances lead to the need for research aimed at establishing baselines that identify the areas of greatest climate risk
\end{abstract}


within nation states. The Argentine Republic is the eighth largest country in the world and the consequences of interannual thermal variability do not affect its territory homogeneously. In this sense, this article intends to identify the interannual thermal anomalies, evidenced in Argentina during the 1982-2012 period, as a basis for the understanding of global warming. The aim is

Recibido: 07 de mayo de 2020

\section{Introducción}

El debate referido al calentamiento global del planeta, entendido como el aumento en el tiempo, de la temperatura media de la atmósfera terrestre y de los océanos (Valencia y Soza, 2005) junto con las consecuencias derivadas del fenómeno, ha abandonado los claustros académicos, para convertirse en una certeza científica y en una creciente preocupación global visibilizada en los Objetivos de Desarrollo Sostenible de la ONU, vinculado específicamente a la acción por el clima (ODS 13).

Simultáneamente, el estudio publicado en 2013 referido a la cuantificación del consenso del calentamiento global en la literatura científica ("Quantifying the consensus on anthropogenic global warming in the scientific literatura", por su título en inglés), ha demostrado que de los 11.944 resúmenes obtenidos de la búsqueda alusiva al término "clima" en el que se examinaron los temas 'cambio de clima global' o 'calentamiento global' comprendidos en el período 19912011; el $66.4 \%$ de los resúmenes no expresaron ninguna posición en torno al calentamiento global antrópico, el 32.6\% aprobó la existencia del fenómeno, $0.7 \%$ lo to establish the main areas of thermal anomalies through the technique of statistical-cartographic analysis, in order to generate basic knowledge about the effects of interannual thermal variability in this South American country.

Keywords: Global warming, interannual thermal variability, temperatures, anomalies, Argentina

Aceptado: 29 de mayo de 2020

rechazó y $0.3 \%$ mantuvo una posición incierta sobre la causa de calentamiento global. (Cook, Nuccitelli, Green et al., 2013, p. 1). Transcurridos más de un lustro desde la publicación del estudio, es probable que las proporciones de las posiciones científica en torno al calentamiento global hayan cambiado.

En tal sentido, algunos autores manifiestan al respecto que, "El Calentamiento Global antropogénico es una realidad, las emisiones de CO2 (Bióxido de Carbono) producto de la quema de combustibles fósiles han alterado el equilibrio climático del planeta." (Arcos Navarro, 2007, p. 11).

El calentamiento global ha sido materia de investigación y discusión desde hace mucho tiempo. Se han buscado diversas explicaciones naturales del fenómeno, pero el tema no pasaba del ámbito científico debido a que, salvo algunos casos aislados de años con clima excepcionalmente frío o cálido, jamás se había ligado el tema a causas antropogénicas. (Power Porto, 2009, p. 102). 
Los sucesos vinculados al "calentamiento" o "enfriamiento" del planeta, per se, no constituyen episodios extraños o anómalos sino que han acompañado la evolución natural del sistema terrestre cuyo principal indicador han sido los glaciares, en sus momentos de expansión (períodos glaciarios) y de contracción (períodos interglaciarios) los cuales han dejado diversos rastros $\mathrm{y}$ evidencias en la geomorfología de los diferentes continentes, fundamentalmente en latitudes altas y en las zonas cordilleranas:

El clima no es estático, sino que a lo largo de la historia de la Tierra ha sufrido variaciones de origen natural, en diferentes escalas temporales, desde decenios a millones de años. Entre las variaciones climáticas naturales mejor conocidas se encuentra el ciclo de períodos glaciales, en el que aproximadamente cada 100.000 años el clima de nuestro planeta es más frío. (Sánchez Sanz, 2007).

A diferencia de los episodios ocurridos con anterioridad, el actual calentamiento global posee dos elementos distintivos: la presencia de la especie humana en el planeta y el hecho de que la principal causa del incremento térmico planetario sea atribuida a sus actividades económicas (modelos productivos):

Las evidencias del clima anómalo y extremo han impactado en todos los sectores de la sociedad -científico, económico, cultural y político-, de tal manera que el entendimiento $y$ experiencia en torno al clima cambiante han sido procesos inherentes tanto a las sociedades menos desarrolladas como a las sociedades industriales avanzadas. (Pinilla, Sánchez et.al., 2012, p. 918).

El resurgimiento del interés ambiental/climático de los últimos 50 años se encuentra estrechamente asociado a los cambios percibidos y experimentados por las sociedades humanas y los impactos económicos, políticos, sociales y culturales derivados. A diferencia de otros períodos climáticos experimentados, por primera vez en la historia, las sociedades contemporáneas poseen pleno conocimiento de los hechos que acontecen: aumento de las temperaturas medias planetarias, regionales y locales, aceleración del ritmo de derretimiento de los cascos glaciares, aumento de la frecuencia e intensidad de los fenómenos climáticos extremos (variabilidad asociada a distintas frecuencias de fenómenos atmosféricosoceánicos), desprendimientos de iceberg de dimensiones extraordinarias, como el generado en la Plataforma de hielo Larsen C de la Antártida Argentina en julio de 2017 con "(...) un espesor de aproximadamente 190 metros y 1.155 kilómetros cúbicos de hielo" (ABC, 2017). En el caso del Cono Sur de América, la República Argentina constituye uno de los países afectados por el calentamiento global (Barros y Camilloni, 2016), presentando escenarios termopluviométricos no alentadores según los estudios realizados al respecto (Banco Mundial, 2014; Reyer et al., 2015). Según el centro de investigaciones del Mar y de la Atmósfera (CIMA-CONICET) en los últimos 50 años el aumento promedio de las temperaturas en el país alcanzó medio grado centígrado, pero en la Patagonia superó $1^{\circ} \mathrm{C}$. (El Cronista, 2016). 
Es por ello que, el presente trabajo dirige sus esfuerzos a contribuir científicamente al conocimiento de la variabilidad térmica interanual, mediante la determinación de las anomalías térmicas en el territorio de la República Argentina en el período 19822012, como línea base de los datos observados durante los últimos 30 años. Estas anomalías, sustentadas en el concepto de anomalías climáticas (Marín, 1990), son entendidas como oscilaciones pronunciadas de los elementos climáticos alrededor de la media estadística, permitiendo la generación de conocimiento científico fiable mediante un método validado, con el fin de apoyar la toma de decisiones fundadas en este país sudamericano.

\section{Marco teórico}

Las anomalías climáticas se definen como la "(...) diferencia entre el valor de un elemento climático en un lugar dado y el valor medio de dicho elemento correspondiente al círculo de latitud del lugar." (Organización Meteorológica Mundial, 2011, p. 13). De acuerdo con la definición formulada, la anomalía mide la 'desviación' del valor observado con respecto a su media climática o histórica (Xavier y Xavier, 1998).
Técnicamente, enfocado desde la terminología climática, la anomalía puede asociarse con el concepto de variabilidad climática, en el sentido de alternancia de registros de los elementos climáticos en torno al valor medio de la serie contemplada. El Grupo Intergubernamental de Expertos sobre el Cambio Climático (IPCC por sus siglas en inglés) define a la variabilidad climática como:

$$
\begin{aligned}
& \text { (...) las variaciones en el estado } \\
& \text { medio y otros datos estadísticos (como } \\
& \text { las desviaciones típicas, la ocurrencia } \\
& \text { de fenómenos extremos, etc.) del } \\
& \text { clima en todas las escalas temporales } \\
& \text { y espaciales, más allá de fenómenos } \\
& \text { meteorológicos determinados. La } \\
& \text { variabilidad se puede deber a procesos } \\
& \text { internos naturales dentro del sistema } \\
& \text { climático (variabilidad interna), o a } \\
& \text { variaciones en los forzamientos } \\
& \text { externos antropogénicos (variabilidad } \\
& \text { externa). (IPCC, 2011, p. 3). }
\end{aligned}
$$

No obstante, se acepta que una anomalía responde a un proceso agudo o pronunciado de dicha variabilidad que resulta excepcional en su ocurrencia y amerita un estudio específico como indicador físico del calentamiento global. 
Fuente: IPCC. Todd Albert, Estados Unidos de América.

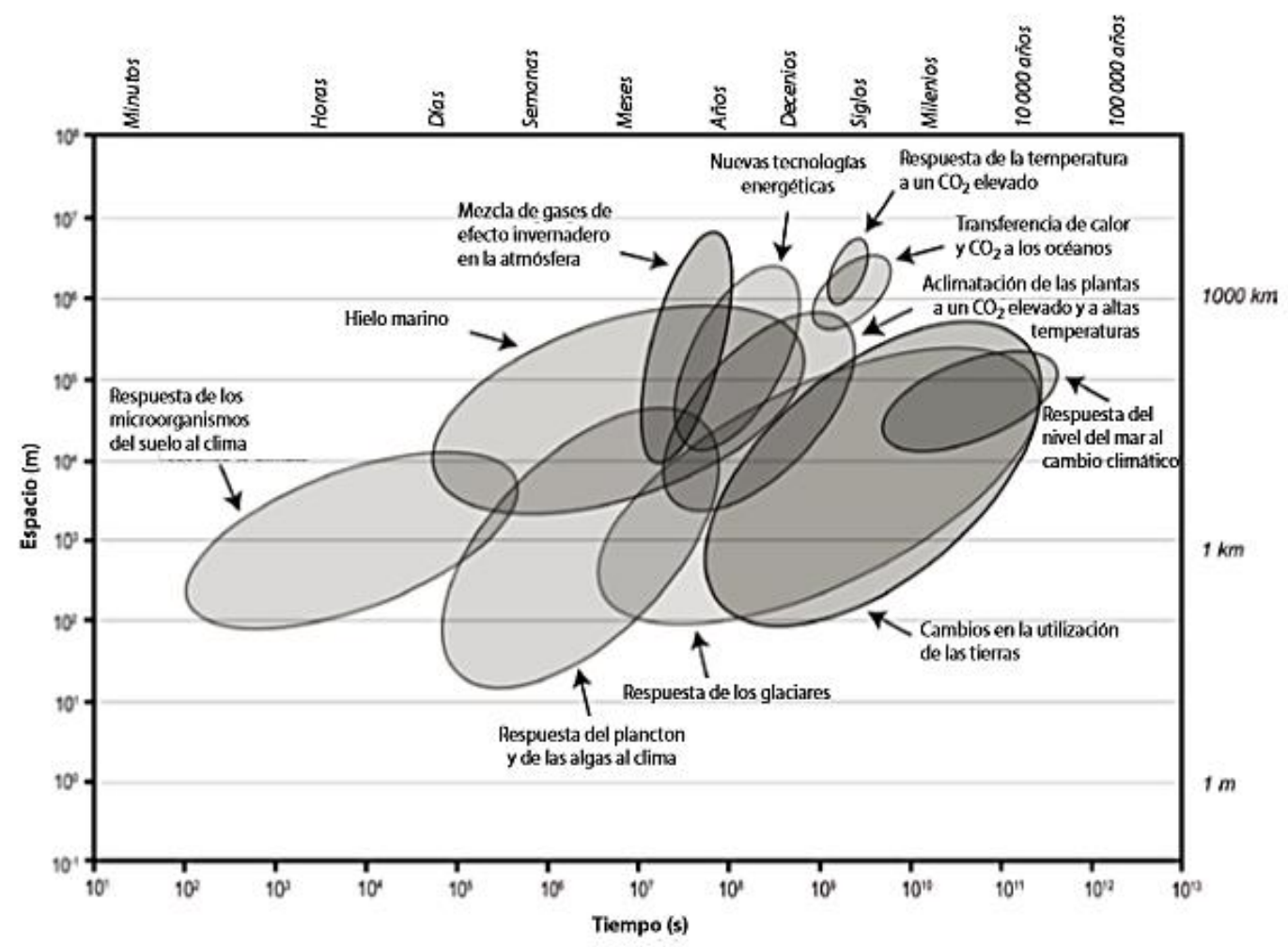

Figura 1. Escalas espaciales y temporales en la ocurrencia de fenómenos climáticos.

En el sistema climático, las interacciones entre los componentes ocurren en todas las escalas espaciales y temporales (Figura 1), tal como lo manifiestan las guías de prácticas climatológicas del IPCC: en la microescala (mayor escala cartográfica) las áreas abarcan aspectos de las características climáticas en zonas pequeñas tales como áreas urbanas y rurales específicas. Un cambio en un microclima puede evidenciar la modificación de las características físicas de la zona:

(...) Las variaciones naturales en un microclima, tales como las relativas al cobijo y la exposición, la insolación y la sombra, también son importantes puesto que pueden determinar, por ejemplo, qué plantas pueden prosperar en un lugar dado o la necesidad de tomar disposiciones sobre la seguridad en el entorno laboral y las actividades de ocio. (IPCC, 2011, p. 6).

La mesoescala abarca el clima de una región de extensión limitada, tal como una cuenca hidrográfica, una conurbación o un bosque. La macroescala (menor escala cartográfica) comprende el clima de vastas zonas geográficas, continentales y planetarias con un mayor rango de generalización.

En tal sentido, a nivel de la macroescala según la clasificación dada, en las últimas décadas las temperaturas planetarias han evidenciado extraordinarias anomalías. Las temperaturas del mes de abril de 2014 han equiparado a las de abril de 2010, como las temperaturas medias más altas desde 1880, según la Administración Nacional Oceánica y Atmosférica de EE. UU. (NOAA, 2014). La 
anomalía térmica presentada en abril de 2014 alcanzó 0,77 grados centígrados por encima del valor normal y se posicionó en el $350^{\circ}$ mes consecutivo con temperaturas más altas respecto al promedio. La última vez que abril fue más frío que el promedio 'normal' fue en 1976. (NOAA, 2014).

Aunque en algunos lugares del planeta, el incremento térmico no ha sido extremo (como en el norte de EE. UU. y el centro de
Canadá, por ejemplo), en otras zonas las temperaturas fueron considerablemente más elevadas de lo habitual. Por ejemplo, en el centro de Siberia la temperatura fue aproximadamente 5 grados más alta del promedio entre los años 1981 y 2010. En la Figura 2 se observan los 9 puntos del planeta donde se registraron anomalías térmicas o pluviométricas en abril del 2014.

Fuente: Administración Nacional Oceánica y Atmosférica de EE. UU. NOAA 2014.

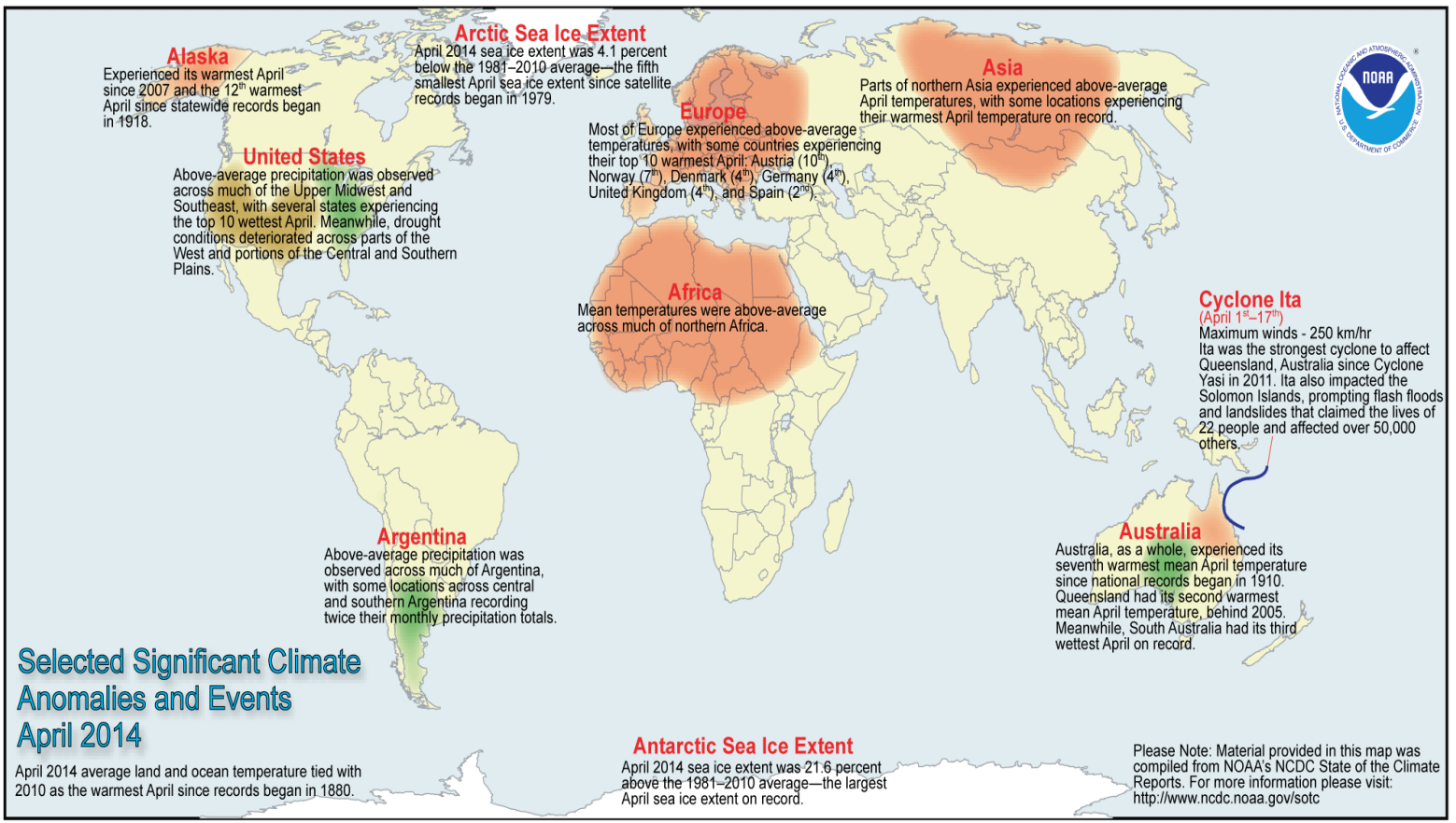

Figura 2. Anomalías y eventos climáticos significativos de abril de 2014.

Las crónicas del momento mencionan que en el océano Ártico se produjo una reducción de la extensión del hielo (pack-ice) del 4,1\% en comparación con la media del período comprendido entre 1981 y 2010. Este aspecto se contrapone con la mayor extensión de hielo jamás observada en el mes abril en el continente antártico: un $21,6 \%$ superior al promedio del período 1981-2010.
El Estado de Alaska vivió su abril más cálido desde el 2007 siendo el $12^{\circ}$ abril más cálido desde el inicio de las mediciones en 1918, mientras que en el resto de los EE. UU. las precipitaciones fueron más abundantes de lo habitual (Tabla 1).

En el caso específico de la República Argentina el índice de precipitaciones fue más alto de lo normal, y en algunas zonas alcanzaron el doble de los valores habituales, 
lo que ha originado una serie de eventos de inundaciones sobre la región de la cuenca del río de la Plata, hacia el este del país, con el desbordamiento de los principales cursos hídricos. Se ha evidenciado a su vez un incremento de los fenómenos extremos de tormentas fuertes y formaciones de tornados.
De las anomalías analizadas se deduce que, los potenciales efectos de las anomalías térmicas positivas en la primavera boreal circumpolar podrían manifestarse con notables incrementos en las precipitaciones de las latitudes medias y anomalías térmicas negativas en la Antártida.

Tabla 1. Anomalías registradas por el satélite NOAA, abril de 2014.

\begin{tabular}{|c|c|c|c|}
\hline \multirow[t]{2}{*}{ REGIÓN } & \multicolumn{3}{|c|}{$\begin{array}{l}\text { ANOMALÍAS REGISTRADAS/ABRIL } 2014 \text { (SATÉLITE } \\
\text { NOAA) }\end{array}$} \\
\hline & Térmicas & Pluviométricas & $\begin{array}{l}\text { Fenómenos } \\
\text { extremos }\end{array}$ \\
\hline $\begin{array}{l}\text { Océano } \\
\text { Ártico }\end{array}$ & $\begin{array}{l}\text { Registros térmicos } \\
\text { más elevados que el } \\
\text { promedio de registros } \\
\text { (anomalías térmicas } \\
\text { positivas) }\end{array}$ & --------- & $\begin{array}{l}\text { Disminución del } \\
\text { Pack-Ice o hielo a } \\
\text { la deriva. }\end{array}$ \\
\hline Alaska & $\begin{array}{l}\text { Registros térmicos } \\
\text { más elevados que el } \\
\text { promedio de registros } \\
\text { (anomalías térmicas } \\
\text { positivas) }\end{array}$ & & $\begin{array}{l}\text { Derretimiento de } \\
\text { Glaciares. } \\
\text { Inundaciones. }\end{array}$ \\
\hline $\begin{array}{l}\text { Norte de } \\
\text { Asia/Siberia }\end{array}$ & $\begin{array}{l}\text { Registros térmicos } \\
\text { más elevados que el } \\
\text { promedio de registros } \\
\text { (anomalías térmicas } \\
\text { positivas) }\end{array}$ & & $\begin{array}{l}\text { Derretimiento del } \\
\text { Permafrost } \\
\text { Inundaciones. }\end{array}$ \\
\hline Europa & $\begin{array}{l}\text { Registros térmicos } \\
\text { más elevados que el } \\
\text { promedio de registros } \\
\text { (anomalías térmicas } \\
\text { positivas) }\end{array}$ & & $\begin{array}{l}\text { Disminución del } \\
\text { Pack-Ice o hielo a } \\
\text { la deriva. } \\
\text { Inundaciones }\end{array}$ \\
\hline $\begin{array}{l}\text { Estados } \\
\text { Unidos de } \\
\text { América }\end{array}$ & 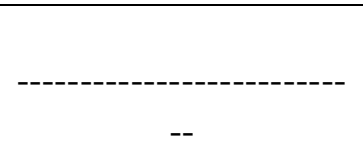 & $\begin{array}{l}\text { Incremento de las } \\
\text { Precipitaciones hacia } \\
\text { el Este del país. }\end{array}$ & Inundaciones. \\
\hline $\begin{array}{l}\text { Norte de } \\
\text { África }\end{array}$ & $\begin{array}{l}\text { Registros térmicos } \\
\text { más elevados que el } \\
\text { promedio de registros } \\
\text { (anomalías térmicas } \\
\text { positivas) }\end{array}$ & ------------------------ & $\begin{array}{l}\text { Sequías extremas } \\
\text { en el África } \\
\text { subsahariana / } \\
\text { Sahel. }\end{array}$ \\
\hline
\end{tabular}




\begin{tabular}{|l|l|l|l|}
\hline Argentina & ------------- & $\begin{array}{l}\text { Incremento de las } \\
\text { Precipitaciones hacia } \\
\text { el Este y sudoeste del } \\
\text { país. }\end{array}$ & Inundaciones. \\
\hline Australia & $\begin{array}{l}\text { Registros térmicos } \\
\text { más elevados que el } \\
\text { promedio de registros } \\
\text { (anomalías térmicas } \\
\text { positivas) }\end{array}$ & $\begin{array}{l}\text { Incremento de las } \\
\text { Precipitaciones hacia } \\
\text { el Este y sudoeste del } \\
\text { país. }\end{array}$ & Inundaciones. \\
\hline Antártida & $\begin{array}{l}\text { Registros térmicos } \\
\text { más bajos que el } \\
\text { promedio de registros } \\
\text { (anomalías térmicas } \\
\text { negativas) }\end{array}$ & $\begin{array}{l}\text { Incremento de las } \\
\text { Precipitaciones }\end{array}$ & $\begin{array}{l}\text { Incremento del } \\
\text { Pack-Ice y de los } \\
\text { glaciares. }\end{array}$ \\
\hline
\end{tabular}

Fuente: Elaboración propia con base en los datos del satélite NOAA, abril de 2014.

Del análisis realizado queda demostrado que el planeta se encuentra atravesando un período de transición, caracterizado por episodios de anomalías meteorológicas y el aumento de ocurrencia de fenómenos meteorológicos extremos, que podrían deberse al incremento térmico sostenido del sistema climático global. La historia climática del planeta demuestra que posterior

\section{Materiales y método}

La presente investigación ha utilizado datos de temperatura media mensual proveniente de 82 estaciones meteorológicas de la República Argentina (sector continental) representadas en la Figura 3, correspondientes al período 1982-2012, provistas por el Servicio Meteorológico Nacional (SMN) dependiente del Ministerio de Defensa de la Nación Argentina. a un periodo de transición en los parámetros de los elementos climáticos, el sistema tiende a establecerse en un nuevo equilibrio dinámico (Acot, 2005), lo que generaría nuevos patrones en la distribución termopluviométrico del planeta impactando de lleno a los ecosistemas y al estilo de vida humano tal como se conoce actualmente.

El periodo en estudio ha sido seleccionado con base en dos criterios principales: la calidad y cantidad de registros térmicos generados y supervisados por el SMN para esta temporalidad y por constituir un período significativo para el análisis de las anomalías térmicas dado que se trata de un lapso en el cual, según los análisis estadísticos previos, se suceden ocurrencias de anomalías positivas y negativas. 
Fuente: Extraído de Fernández-Long, M. et.al 2016

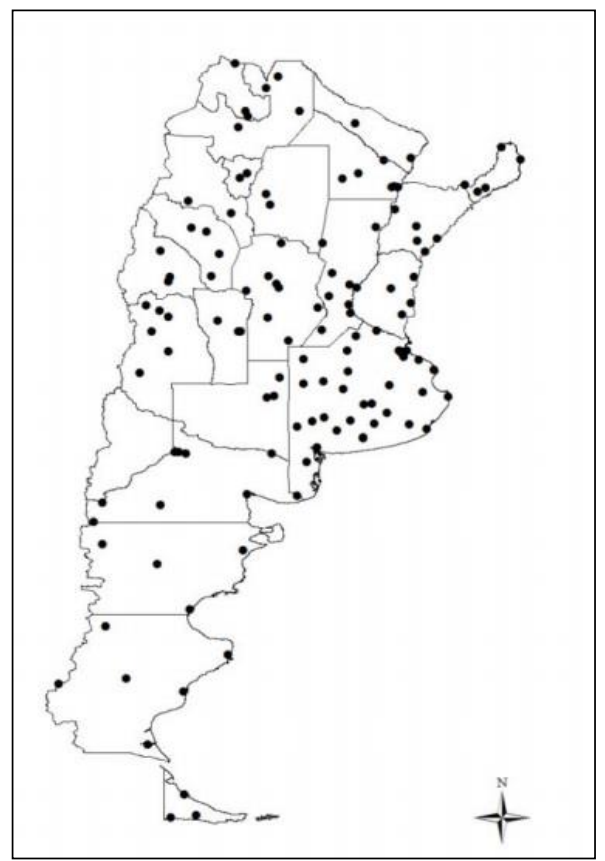

Figura 3. Distribución geográfica de las Estaciones Meteorológicas.

A partir de los registros de temperaturas medias mensuales, previo análisis de tendencias u homogeneidad, se han calculado las anomalías de temperatura que expresan el comportamiento térmico de un mes o de la media anual en relación con el dato promedio de la serie, considerado normal, para dicho mes o año. El indicador resulta de restar a la temperatura media del mes o año objeto de cálculo, la temperatura media de dicho mes o del año para el período considerado: 19822012.

La fórmula es la siguiente: $A T_{i}=T_{i}-\bar{T}_{i}$

Donde:

$A T_{i}$ : anomalía de la temperatura del mes $i$

$T_{i}$ : temperatura media mensual del mes $i$

$\bar{T}_{i}$ : temperatura media del mes $i$ en el período 1982-2012.

Los resultados se expresan en grados centígrados $\left({ }^{\circ} \mathrm{C}\right)$. Cuando los valores resultantes del indicador son positivos, muestran temperaturas superiores a las normales en ese mes o año, mientras que si los resultados son negativos denotan temperaturas inferiores a las del promedio de la serie. En esta oportunidad se han obtenido a partir de los datos y de la aplicación de la fórmula mencionada, las anomalías térmicas anuales para la República Argentina en el período 1982-2012.

Finalizada la etapa anterior, se procedió a representar la información obtenida en gráficos lineales, con la correspondiente línea de tendencia polinómica. Estas representaciones han sido elaboradas sólo, para aquellas estaciones meteorológicas que contuvieran la serie de datos completa, es decir, sin meses faltantes ni meses incompletos dado que, esta circunstancia, imposibilita el cálculo del promedio mensual. Por lo tanto, de las 82 estaciones con información meteorológica, sólo 12 reúnen 
tales requisitos para la elaboración de los gráficos mencionados.

Simultáneamente, se elaboró la cartografía en formato vectorial, de tipo isoplético, lo que posibilitó la visualización de las características de la distribución espacial de las anomalías térmicas anuales. En este sentido, se ha trabajado con el software propietario de la empresa ESRI: el ArcGis 10.1 para la confección de los mapas, utilizando el procedimiento de interpolación para generar las isanómalas, que son curvas que, en una representación cartográfica, unen los puntos del espacio geográfico con la misma anomalía térmica en relación con el valor medio de la serie. El método de interpolación que se aplicó fue el IDW (Ponderación de distancia inversa), herramienta del ArcMap que estima los valores de las celdas calculando promedios de los valores de la muestra en la vecindad de cada celda de procesamiento. Se ha utilizado el método de ponderación de distancia inversa por ser flexible, permitiendo identificar "barreras" en la distribución espacial de los datos y ajustar los resultados a la energía de la superficie. Cuanto más cerca está un punto del centro de la celda que se está estimando, más influencia o peso tendrá en el proceso de cálculo del promedio. Se incluye las anomalías térmicas anuales a fin de dimensionar cuali-cuantitativamente el fenómeno de Anomalías térmicas.

\section{Resultados}

Si se observa el gráfico de anomalías térmicas del planeta (1850-2016) elaborado a partir del registro global de la temperatura, compilado por Climatic Research Unit de la Universidad de East Anglia (Figura 4), es posible advertir varios aspectos: en primer lugar, hasta el año 1940 el planeta experimentó un período de anomalías térmicas negativas, con temperaturas promedio inferiores al de la serie en estudio, con tan solo 1 año (1876) que denota anomalía térmica positiva. Desde la década del '40 hasta la del '70, se advierte una etapa transicional en la que se intercalan años, en su mayoría, con anomalías térmicas negativas y otros con anomalías térmicas positivas. Desde la década de 1980 en adelante, la mayoría de los años muestran anomalías térmicas positivas, aspecto que, como se ha mencionado previamente, ha sido una de las razones que motivó la selección del período de estudio para la presente investigación.

Cabe resaltar que, para la década de 1980, se advierte la misma tendencia transicional identificada en la etapa anterior, pero con la diferencia de que, en esta década, predominan las anomalías térmicas positivas para los años que la componen. 
Fuente: Climatic Research Unit de la Universidad de East Anglia 2018.

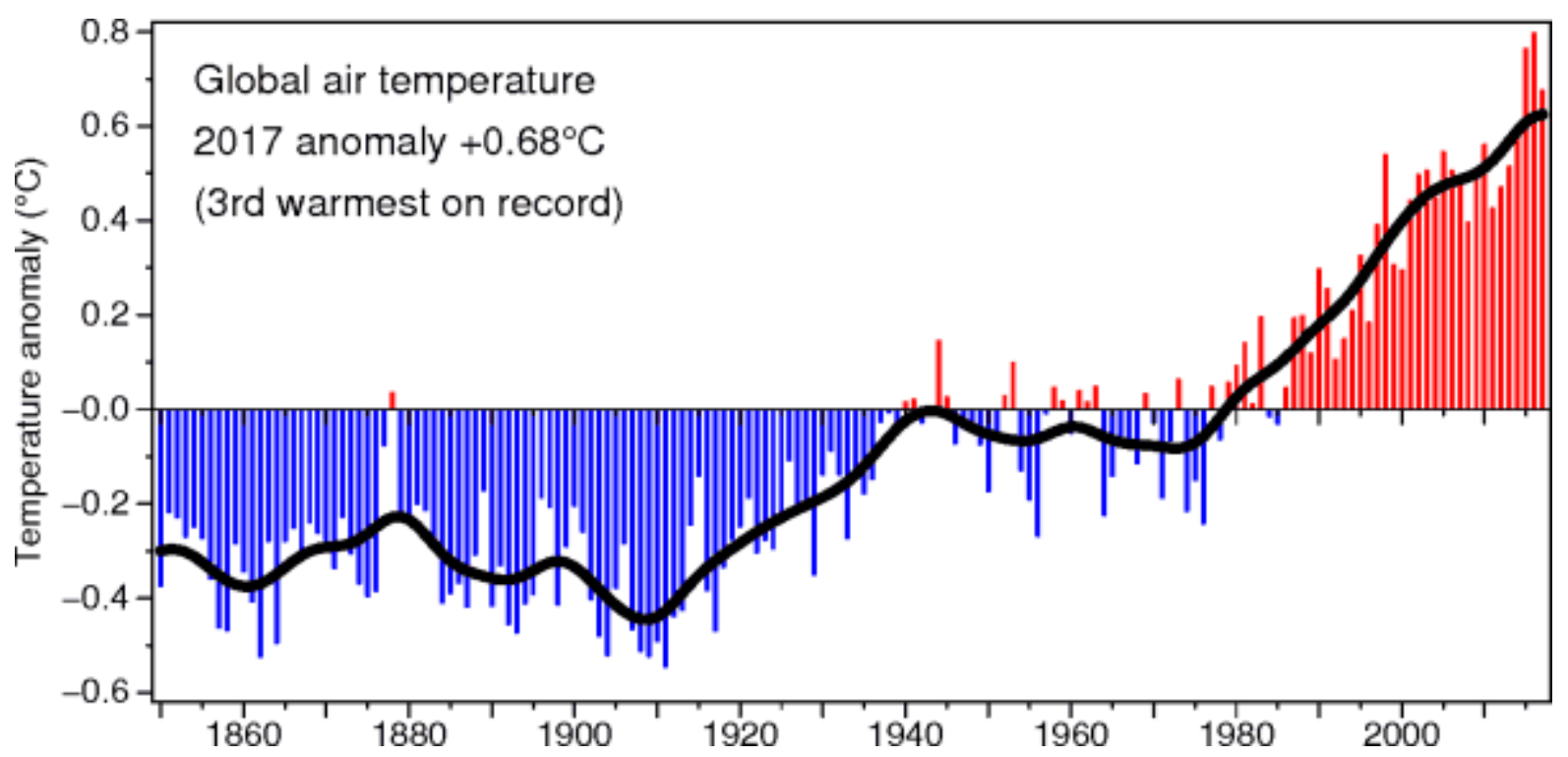

Figura 4. Registro global de la temperatura desde 1850 hasta 2017.

Al observar los gráficos que se muestran en la Figura 5 (a y b) se advierte que, para las 12 estaciones meteorológicas en las que se representó la evolución de las anomalías térmicas durante el período de estudio, existe una tendencia polinómica ascendente del indicador en los últimos 30 años; especialmente en las estaciones de Córdoba Aero, Ceres Aero y Laboulaye Aero -todas ubicadas en el centro de Argentina- donde la línea de tendencia polinómica adquiere un aumento considerable. Por su parte, Salta Aero, Formosa Aero y Junín Aero son las únicas cuyas tendencias son estables, incluso es posible advertir un leve descenso.

María Emilia Pérez (2006), al estudiar las variaciones térmicas en el norte argentino, manifiesta que "la temperatura no presenta un aumento continuo y sostenido [...], sino que, por el contrario, se advierte una alternancia de décadas más cálidas con otras más frías" ( $\mathrm{p}$. 25). Esta alternancia se ratifica en la totalidad de los gráficos, evidenciándose la misma cantidad de años con anomalías positivas y negativas. En tal sentido, los gráficos reflejan a una mayor escala espacio-temporal, los aspectos analizados en los registros globales de temperatura 1850-2017, pero con menor grado de generalización, brindando mayor relevancia a las oscilaciones mensuales acaecidas en el período.

La mayoría de los años en estudio presentan máximos valores de anomalías positivas próximos a $1^{\circ} \mathrm{C}$, a excepción de Ceres Aero que en el año 2012 registra $1,35^{\circ} \mathrm{C}$ más que la media del período de estudio. Por su parte, las mayores anomalías negativas también alcanzan una diferencia de alrededor de $1^{\circ} \mathrm{C}$ en todos los casos, excepto en Salta Aero y en Formosa Aero, donde los guarismos rondan los 0,5 y $0,6^{\circ} \mathrm{C}$, respectivamente. La estación meteorológica de Buenos Aires presenta una marcada anomalía positiva, influida probablemente por la isla de calor generada por la presencia de la ciudad. Atendiendo estas características en el comportamiento de las anomalías térmicas es posible determinar, a priori, el paralelo de $35^{\circ}$ sur como límite o 
zona de cambio de tendencias de las anomalías según la ubicación geográfica de las estaciones meteorológicas en estudio.

Hacia el norte de los $35^{\circ}$ sur, las estaciones meteorológicas denotan anomalías térmicas moderadas, más leves y próximas a los valores promedios de la serie en estudio. Por su parte las estaciones meteorológicas ubicadas hacia el sur del paralelo de $35^{\circ}$ sur acentúan el comportamiento en las anomalías térmicas tanto positivas como negativas, lo que remite a la clásica relación entre la temperatura y la latitud y su influencia en la configuración del campo térmico de superficie.

Fuente: Elaboración propia a partir de los datos proveídos por el SMN, 2014.

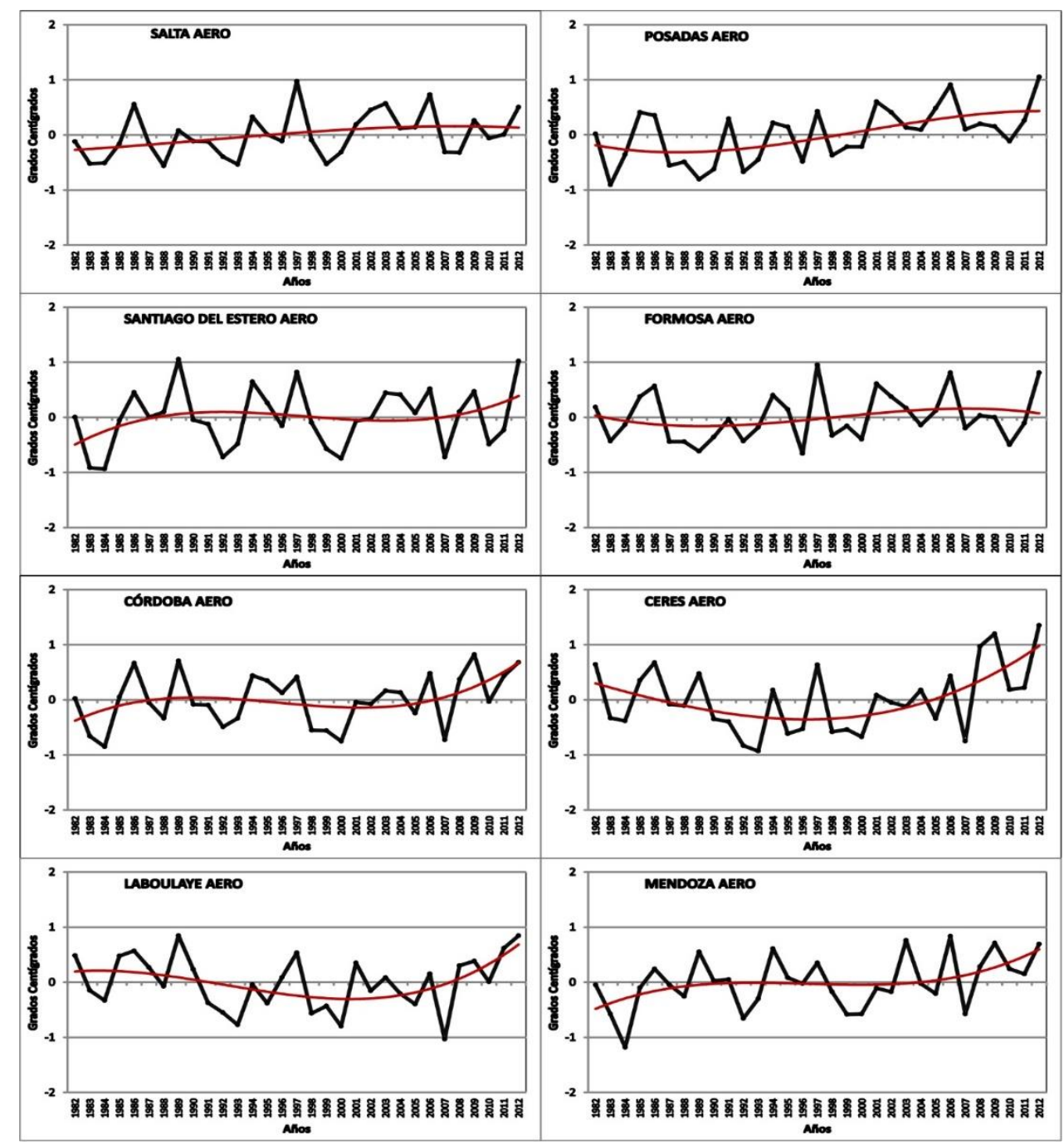

Figura 5 (a). Anomalías térmicas anuales durante el período 1982-2012, en estaciones con series meteorológicas completas. 
Fuente: Elaboración propia a partir de los datos proveídos por el SMN, 2014.

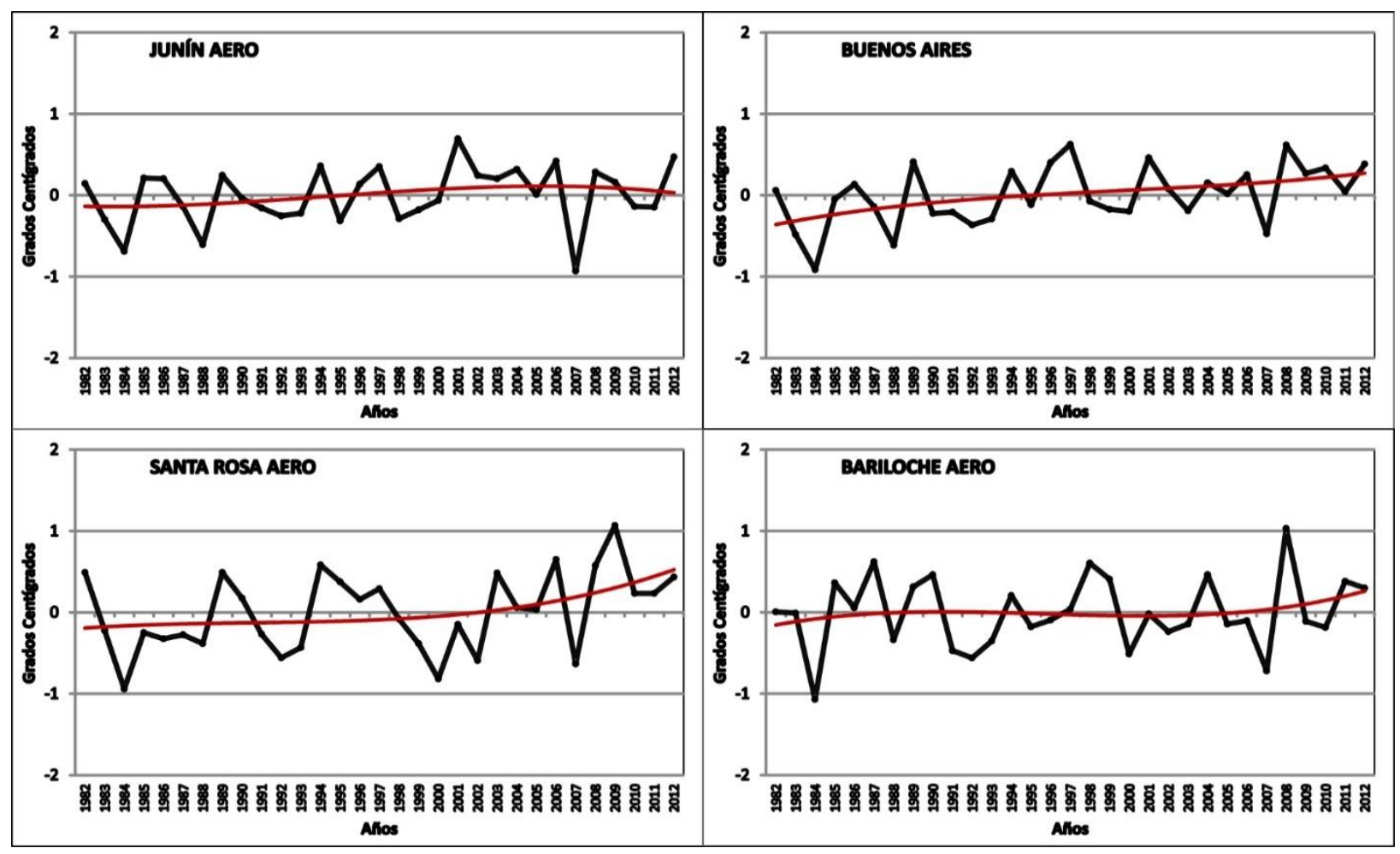

Figura 5 (b). Anomalías térmicas anuales durante el período 1982-2012.

Si bien, conforme a lo expresado por Xavier y Xavier (1998, p. 36), el análisis ideal de anomalías térmicas sería aquel que se realizaría sin el efecto altitudinal, por ejemplo, con las temperaturas de la superficie del mar en la cuenca del Atlántico (entre $30^{\circ}$ $\mathrm{N} \mathrm{y} 30^{\circ} \mathrm{S}$ ); el interés geográfico gira en torno a la presencia humana y los espacios ecuménicos por lo que el análisis de las anomalías térmicas resulta imprescindible en las áreas continentales siempre, mediados por los ajustes y controles pertinentes para tales efectos.
En este sentido, las representaciones cartográficas de la Figura 6 (a, b, c, y d), compuesta por 31 mapas, uno por cada año del período analizado, representan a las isanómalas de temperatura (en grados centígrados) del sector continental de la República Argentina, para el período 19822012. Todos los documentos cartográficos generados tienen como fuente a los datos provistos por el Servicio Meteorológico Nacional. En la última página, junto al mapa correspondiente al año 2012, se ubican las referencias que son válidas para toda la cartografía. 

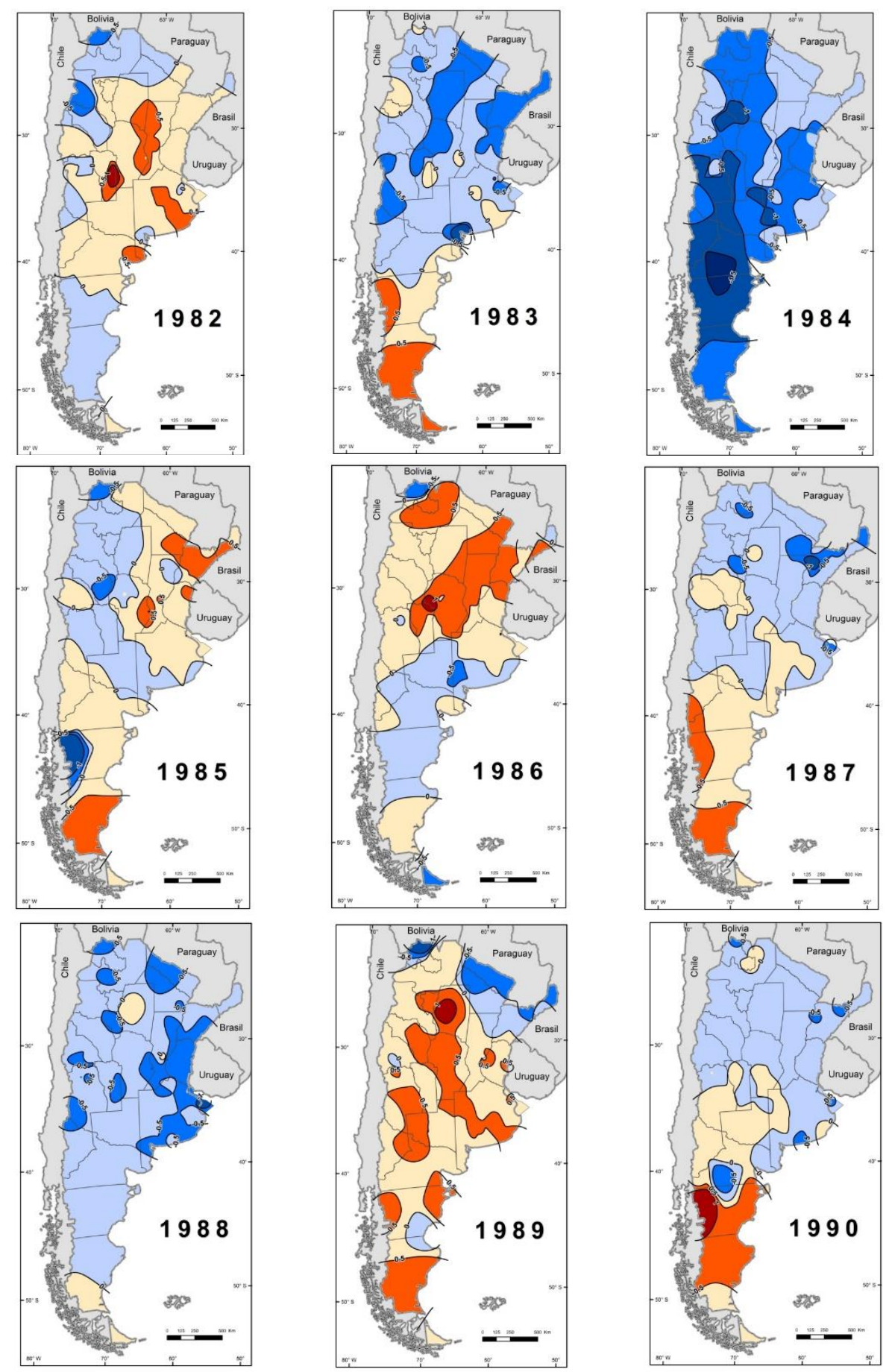

Figura 6 (a). Anomalías térmicas anuales para cada año del período 1982-1990. 

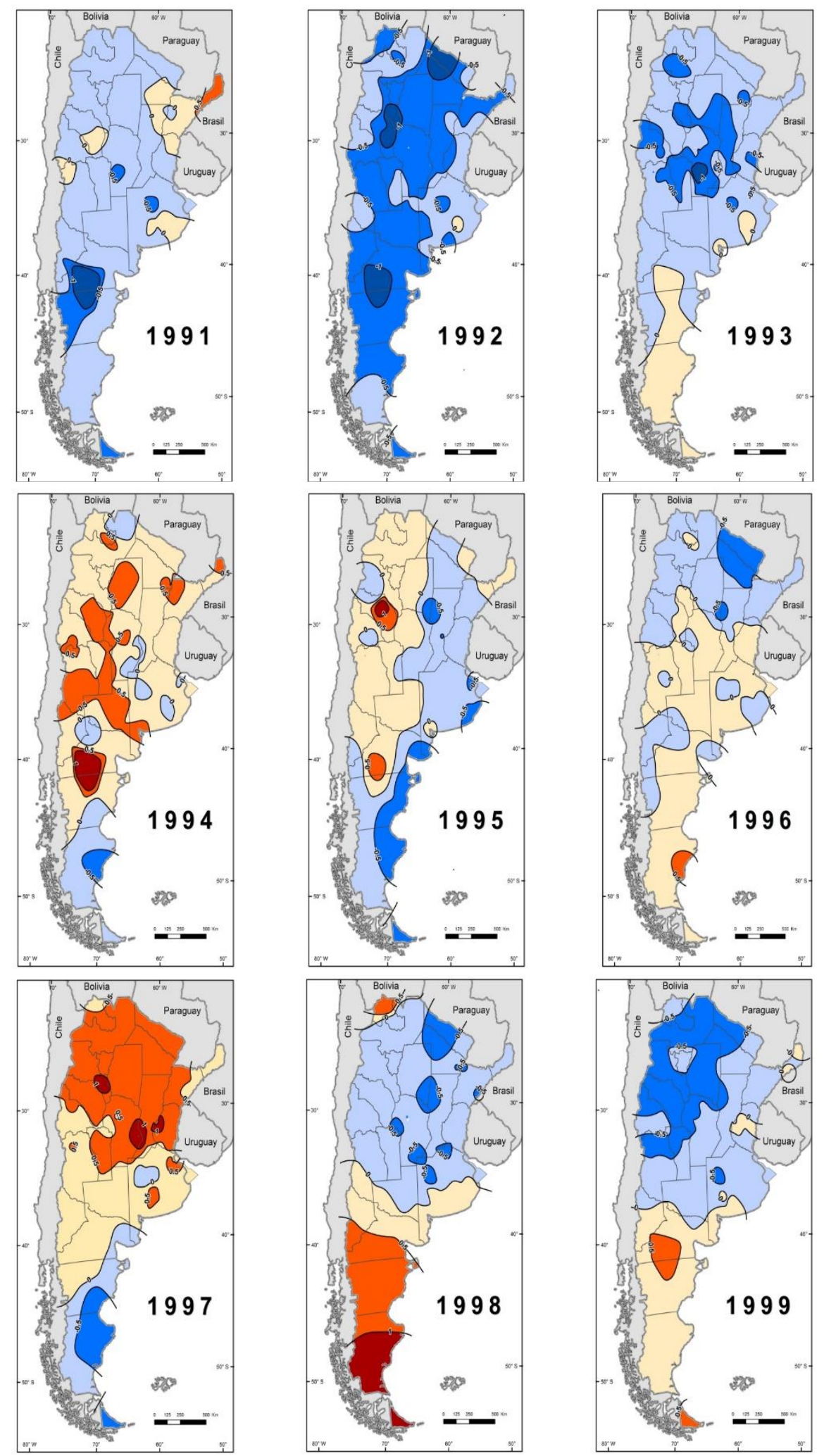

Figura 6 (b). Anomalías térmicas anuales para cada año del período 1991-1999. 

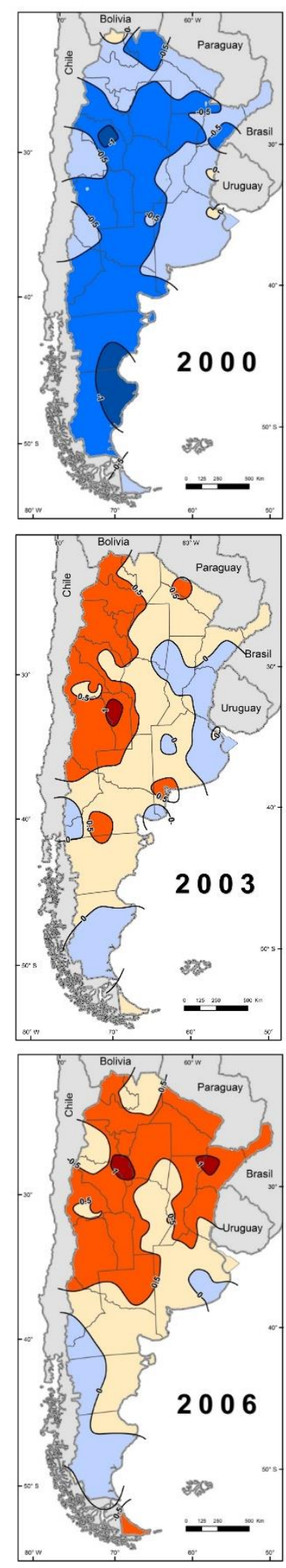
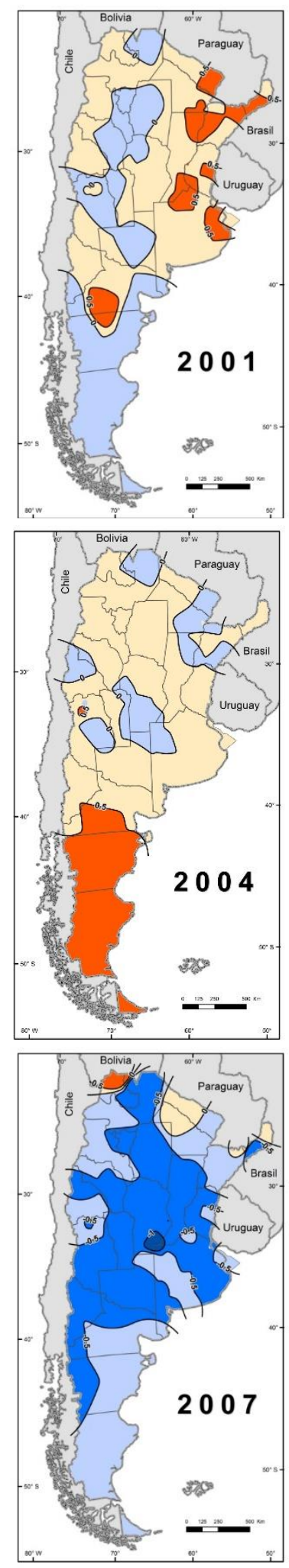
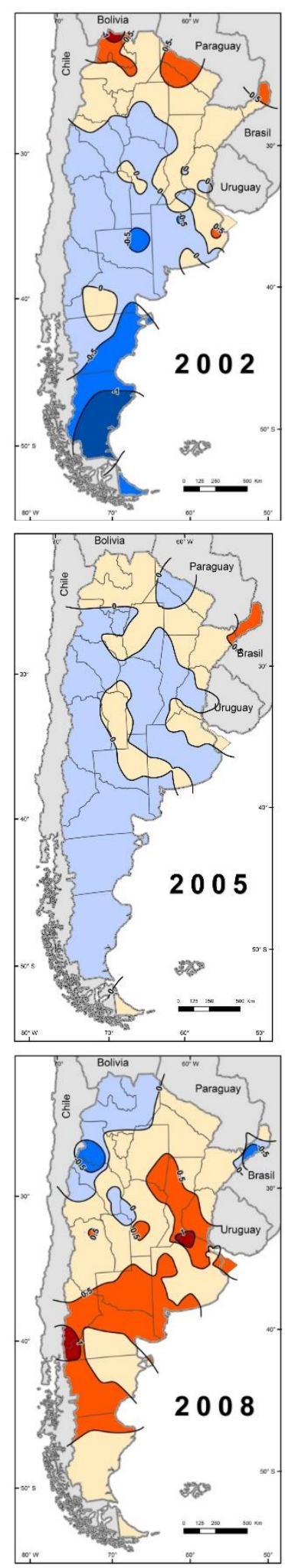

Figura 5 (c). Anomalías térmicas anuales para cada año del período 2000-2008 

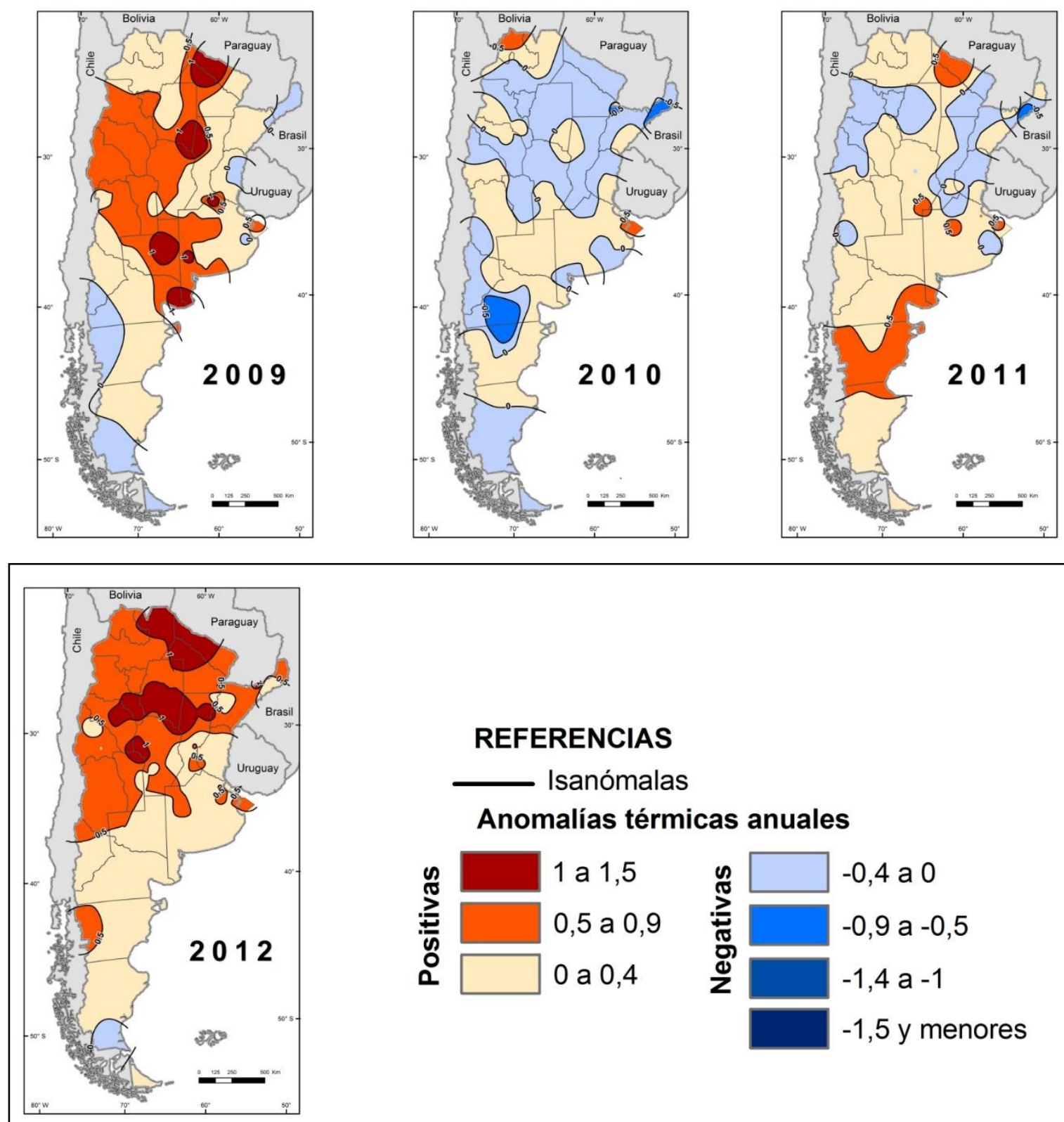

\section{REFERENCIAS}

Isanómalas

Anomalías térmicas anuales
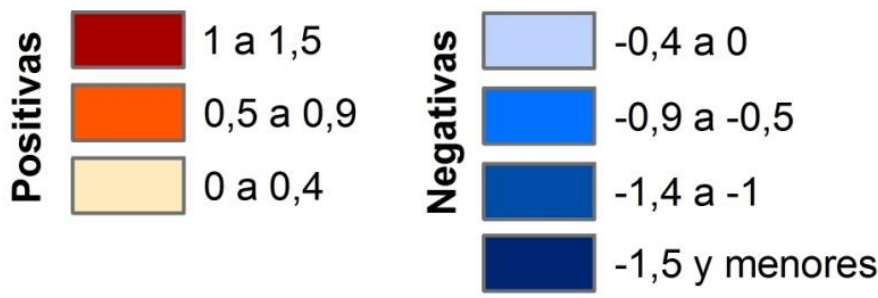

Figura 5 (d). Anomalías térmicas anuales para cada año del período 2009-2012.

Fuente: Elaboración propia a partir de los datos proveídos por el SMN, 2014.

En una primera instancia de análisis, no se advierte un aumento homogéneo de temperaturas en el país en los últimos años, sino una alternancia entre años fríos (1984, 1988, 1992, 1993 y 2000) en los que el territorio nacional tiene anomalías negativas; años cálidos, tales como el 2012, con anomalías térmicas positivas en toda la superficie argentina; y años donde el calentamiento es diferencial, regional, localizado y ubicuo. 
En este sentido, si se analizan, por ejemplo, los mapas correspondientes a los años 1998 y 1999 se identifica un proceso de enfriamiento en el centro y norte del país mientras que la Patagonia eleva sus temperaturas; y, por el contrario, en 1997 es la región patagónica quien se encuentra más fría y el centro y norte del país se ha calentado; lo que advierte sobre la existencia de ciertos mecanismos de "compensación térmica" en el espacio argentino, denotando los esfuerzos del sistema climático regional por lograr un equilibrio dinámico.

Casos excepcionales en la serie analizada han sido los ocurridos en los años 1984, 1992, 2000 y 2007, en donde se han producidos eventos con altos valores de isanómalas negativas que se extendían por la región de Cuyo en el oeste argentino y por el norte y centro Patagónico. Por el contrario, los años 1989, 1997, 2003, 2006, 2008, 2009 y 2012, representan períodos con predominios de anomalías térmicas positivas en todo el territorio argentino.

Si correlacionamos el análisis cartográfico con la sucesión de los eventos registrados del fenómeno ENOS es posible llegar a la explicación del surgimiento de las anomalías térmicas en el territorio argentino:

Durante el siglo XX y hasta antes de El Niño extraordinario de 1997/98, ocurrieron unos 25 episodios El Niño de diferente intensidad. Las referencias bibliográficas indican que los eventos El Niño de 1891 y 1925, fueron eventos de intensidad comparable a los de 1982/83 y 1997/98. En lo que va del siglo XXI, de acuerdo al índice ONI (Oceanic Niño Index) de la NOAA, se han presentado cuatro episodios El Niño en el Pacífico central: dos de intensidad débil (años 2004/05 y 2006/07) y dos de intensidad moderada (años 2002/03 y 2009/10). (SENAMHI, 2014, pág. 24).

Cabe destacar el episodio registrado en el año 2012, no sólo porque la anomalía térmica positiva abarca de manera generalizada a todo el territorio nacional, sino también porque, la magnitud de las anomalías positivas que ha afectado al país sudamericano ha oscilado entre 0,5 a $1,5^{\circ} \mathrm{C}$ que resultan significativas para las latitudes medias.

Barros y Camilloni (2016) corroboran lo mencionado cuando se refieren al incremento de la temperatura media anual en superficie de la Argentina y sostienen que se ha experimentado "(...) un aumento de temperatura promedio de medio grado entre 1960 y 2010, con calentamientos mayores en sólo algunas zonas e incluso con enfriamientos en un eje central desde el noreste de la Patagonia hasta Santiago del Estero" (p. 169).

Este aumento fue menor al del promedio global de las áreas continentales del planeta debido, probablemente, a la fuerte influencia moderadora de los océanos del hemisferio sur que se hace evidente en la menor variación que poseen los datos térmicos registrados en Argentina (Barros y Camilloni, 2016, p. 169; Pérez, 2006, p. 25).

En conclusión, las anomalías térmicas registradas en el territorio de la República Argentina, para el período 1982-2012, denotan a diferencia de las etapas del comportamiento de anomalías planetarias, la prolongación de la etapa transicional al menos hasta la segunda mitad de la década del '10 del siglo XXI, probablemente asociada a la menor proporción continental del 
hemisferio sur y a la moderación térmica causada por el predominio oceánico.

\section{Conclusiones}

Si se observa la evolución temporal de las anomalías térmicas registradas en la República Argentina a lo largo del período de análisis (1982-2012) es posible advertir que:

1) La temperatura no presenta un aumento continuo y sostenido desde 1982 hasta 2012 sino que, por el contrario, se advierte una alternancia de períodos fríos y de períodos cálidos, debido a que se contabilizan la misma cantidad de años con anomalías positivas y negativas.

2) Existe una marcada tendencia polinómica al aumento de las anomalías térmicas positivas en los últimos años, especialmente en las estaciones meteorológicas ubicadas en el centro del país.

Si se analiza la distribución espacial de las anomalías en el territorio argentino:

- $\quad$ No hay evidencia de un calentamiento homogéneo en los últimos años, sino que existen regiones cálidas y regiones frías simultáneamente, a excepción de algunos años, como 2012, en el cual, todo el territorio argentino ha sido afectado por anomalías térmicas positivas.

- Los años fríos han evidenciado anomalías mayores en grados centígrados que los registrados en los años cálidos.

- $\quad$ Se han registrado un equilibrio en la sucesión de años fríos: 1984, 1992, 2000 y 2007 (anomalías térmicas negativas en todo el país) y años cálidos: 1989, 1997, 2003, 2006, 2008, 2009 y 2012, (anomalías térmicas positivas en todo el territorio nacional).
- En la mayoría de los años que componen el período en estudio, el calentamiento y enfriamiento es diferencial, es decir que, mientras que en una región se incrementan los valores térmicos, en otras descienden.

- Los valores de las isanómalas, en general, oscilan entre $1^{\circ} \mathrm{C} \mathrm{y}-1^{\circ} \mathrm{C}$ en casi todos los años y en prácticamente toda la extensión del país, incluso en 1996 y 2005 las anomalías registradas no superaron el medio grado centígrado. La excepción ocurrió en 1984 y 2012, con valores altos de anomalías térmicas negativas y positivas, respectivamente.

- $\quad$ De modo general, se puede establecer en el paralelo de $35^{\circ}$ sur, el límite o zona de cambio en las tendencias y comportamientos de las anomalías térmicas, siendo más moderadas hacia el norte del paralelo y más acentuadas hacia el sur de este.

Por todo lo mencionado, no resulta aventurado afirmar que el análisis de las isanómalas en el territorio de la República Argentina evidencia la activación de mecanismos de "compensación térmica" regional, por el cual el sistema climático, tiende a lograr un equilibrio; ya sea para mantener los patrones actuales de comportamiento en los elementos climáticos o ya sea para arribar a otro equilibrio en los mismos.

El estudio de las anomalías térmicas como indicador de variabilidad térmica interanual, resulta pertinente, no solo para corroborar, ratificar y delimitar la magnitud de este fenómeno planetario, sino también, para analizar las correlaciones entre el incremento térmico y los comportamientos de los demás elementos climáticos (precipitaciones, 
presión atmosférica, humedad, vientos). Simultáneamente, a la par de las anomalías térmicas como indicadores del calentamiento global, resulta necesaria la generación y consideración de otros indicadores, como las anomalías pluviométricas y fenómenos extremos, a fin de obtener una caracterización integral del fenómeno en estudio.

\section{Referencias bibliográficas}

Acot, P. (2005). Historia del clima. Desde el Big Bang a las catástrofes climáticas. Buenos Aires: El Ateneo.

Arcos Navarro, G. (2007). Calentamiento Global. Revista Ciencia UAT. Vol. 2 (2): 10-14. ISSN 2007-7521. México. Disponible en: https://www.redalyc.org/pdf/4419/441 942910004.pdf

Barros, V., y Camilloni, I. (2016). La Argentina y el Cambio Climático. De la física a la Política. Universidad de Buenos Aires, Ciudad Autónoma de Buenos Aires: EUDEBA.

Climatic Research Unit. (2017). Registro global de la temperatura y anomalías desde 1850 hasta 2017. Disponible en: http://www.cru.uea.ac.uk/

Cook J., Nuccitelli D., Green S., Richardson M., Winkler, B., Painting R., Way R., Jacobs P., y Skuce A. (2013). Quantifying the consensus on anthropogenic global warming in the scientific literatura. Environmental Research Letter. 8 (2013) 024024 (7pp). $\quad$ DOI: $10.1088 / 1748$ 9326/8/2/024024

Fernández-Long, M., Barnatán, I., Dominici, C., y Murphy, G. (2015). Información agroclimática de las heladas en la
Argentina: generación y uso. Revista Meteorológica. Volumen 41- Número 2. pp. 7-32. Buenos Aires. Disponible en:

http://www.meteorologica.org.ar/wpcontent/uploads/2017/03/RevistaMete orologica_Vol42N2.pdf

Marin, M. (1990). Normalidad y anomalía en la investigación climatológica actual. Revista de Geografía, vol. XXIV. Barcelona, pp. 35-41.

National Oceanic and Atmospheric Administration (NOAA). (2014). Global Climate Report - April 2014. Disponible en: https://www.ncdc.noaa.gov/sotc/global /201404

Panel Intergubernamental de Expertos sobre el Cambio Climático (IPCC). (2011). Guía de prácticas climatológicas. Organización Meteorológica Mundial (OMM) M-N ${ }^{\circ}$ 100.-Naciones Unidas.

Pérez, M. (2006). Fluctuaciones climáticas y variabilidad temporal del clima en el Norte Argentino - 1931/2005. Revista Geográfica Digital, Año 3- $N^{\circ} 6$. Disponible en: http://hum.unne.edu.ar/revistas/geowe b/homeig0.htm

Periódico ABC España. "El iceberg más grande del mundo ya es una realidad". Publicado: 07/07/2017. Consultado 10 de marzo de 2020. Disponible en: http://www.abc.es/sociedad/abciiceberg-mas-grande-mundo-realidad201707062145_noticia.html

Pinilla Herrera, M., Sánchez, J., Rueda, A., y Pinzón, C. (2012). Variabilidad climática y cambio climático: percepciones y procesos de adaptación espontánea entre 
campesinos del centro de Santander, Colombia. Revista de la Asociación Española de climatología. Madrid. Disponible en: http://aeclim.org/wpcontent/uploads/2016/02/0090_PU-SAVIII-2012-MC_PINILLA.pdf

Power Porto, G. (2009). El calentamiento global y las emisiones de carbono. Revista Ingeniería Industrial, núm. 27, pp. 101-122. Disponible en: https://www.redalyc.org/pdf/3374/337 428493007.pdf

Sánchez Sanz, A. (2007). Cambio climático: situación actual y oportunidades para la acción. Real Academia de Ciencias Veterinarias. Área de Gobierno de Medio Ambiente y Servicios a la Ciudad. Excmo. Ayuntamiento de Madrid-España. Disponible on line en:

http://racve.es/publicaciones/cambio- climatico-situacion-actual-yoportunidades-para-la-accion/

Servicio Nacional de Meteorología e Hidrología del Perú - SENAMHI (2014). El Fenómeno El Niño en el Perú. Ministerio del Ambiente MINAM. República del Perú. Disponible en: http://www.minam.gob.pe/wpcontent/uploads/2014/07/Dossier-ElNi\%C3\%B1o-Final_web.pdf

Valencia, M., y Soza, M. (2005). Definiciones Climáticas. Disponible en:

https://www.cec.uchile.cl/ mivalenc/de finicion.htm

Xavier, T., y Xavier, A. (1998). Cálculo de anomalias na pesquisa climática: usos e abusos. X CBMET - Congresso Brasileiro de Meteorologia e VIII Congresso da FLISMET / BRASÍLIA, Octubre de 1998. 